\title{
Tres meses de rehabilitación interdisciplinaria incrementa la fuerza muscular respiratoria de escolares obesos
}

\author{
Three months of interdisciplinary rehabilitation increases respiratory muscle strength \\ in obese schoolchildren

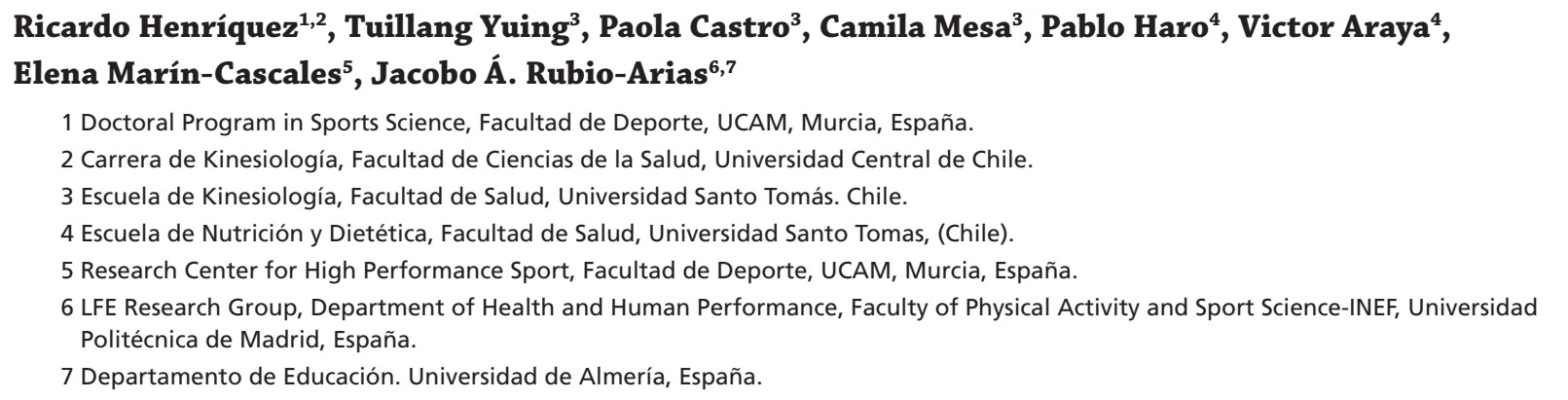

CORRESPONDENCIA:

Ricardo Andrés Henríquez Flores

r.henriquez.f@gmail.com

Recepción: agosto 2020 • Aceptación: febrero 2021
CÓMO CITAR EL ARTÍCULO:

Henríquez, R., Yuing, T., Castro, P., Mesa, C., Haro, P., Araya, V., Marín-Cascales, E., Rubio-Arias, J., (2021). Tres meses de rehabilitación interdisciplinaria incrementa la fuerza muscular respiratoria de escolares obesos. Cultura, Ciencia y Deporte, 16(49), 425-432. http://dx.doi.org/10.12800/ccd.v16i49.1544

\section{Resumen}

Actualmente se desconocen los efectos de un programa interdisciplinario, basado en entrenamiento físico, sobre la fuerza muscular respiratoria (FMR) de escolares obesos. El objetivo es analizar los efectos de un programa de intervención interdisciplinario de 3 meses de duración sobre la masa adiposa (MA), masa muscular (MM) y la FMR en escolares con obesidad infantil y sin patología respiratoria. 11 escolares, 7 de género masculino y 4 de género femenino, participaron durante 3 meses del programa interdisciplinario, atendidos por nutricionistas, psicólogos y entrenadores. Se evaluó FMR con pimometría y composición corporal (CC) con antropometría. Se observó el aumento significativo de la FMR expresada en presión inspiratoria máxima (PIM) $(p=$ $0.029)$ y presión espiratoria máxima (PEM) $(p=0.006)$ en relación con sus valores de referencia y valores absolutos. Se observó una disminución significativa en el IMCZ score $(p=0.04)$ y se encontraron asociaciones significativas entre PIM (PIM/MA $p=0.005 ; r=-0.769$ ) y PEM (PEM/MM $p=0.001 ; r=0.835$ ) con variables de la CC. En conclusión, 3 meses de tratamiento interdisciplinario de la obesidad infantil evocan incrementos en la PIM y PEM y estos incrementos se asocian a variaciones en la CC.

Palabras clave: obesidad pediátrica, músculos respiratorios, rehabilitación, presiones respiratorias.

\begin{abstract}
At present, there is no evidence regarding the effects of an interdisciplinary program, based on physical training, on respiratory muscle strength (FMR) in obese schoolchildren. The objective was to analyze the effects of a three-month interdisciplinary intervention program on fat mass (MA), muscle mass (MM) and FMR in schoolchildren with childhood obesity and without respiratory pathology. 11 schoolchildren, seven males and four female, participated during three months of the interdisciplinary program. Supervised by nutritionists, psychologists and trainers. FMR was evaluated with pimometry and body composition (CC) with anthropometry. There was a significant increase in FMR, expressed as maximum inspiratory pressure (PIM) $(p=0.029)$ and maximum expiratory pressure (PEM) $(p=0.006)$ in relation to their reference and absolute values. A significant decrease in the BMI score was found ( $p=0.04)$, as well as associations between PIM (PIM / MA p = 0.005; $r=-0.769$ ) and PEM (PEM / MM p $=0.001 ; r=0.835)$ with variables of CC. In conclusion, three months of interdisciplinary treatment of childhood obesity evoke increases in PIM and PEM, and these increases are associated with variations in CC.
\end{abstract}

Key words: pediatric obesity, respiratory muscles, rehabilitation, respiratory pressures. 


\section{Introducción}

La obesidad infantil (OI) es un problema global que ha crecido en prevalencia desde 1980 hasta la fecha actual. Hasta el año 2015, existían 107.7 millones de niños con obesidad, correspondiendo a un 5\% de la población infantil (GBD 2015 Obesity Collaborators et al., 2017). En este sentido, la OI puede repercutir de manera negativa en el desarrollo de diferentes sistemas. En el caso del sistema respiratorio, se sabe que la obesidad puede aumentar la expresión de fenotipos asmáticos (Scott et al., 2017; Wadden et al., 2018) y que deteriora las presiones máximas respiratorias en escolares (da Jung \& Schivinski, 2014) variando su función pulmonar (Forno et al., 2018). Existe una asociación entre el asma y la obesidad, donde se le puede asignar el rol de causa/efecto a ambas patologías, describiendo que la obesidad es un factor de riesgo que puede producir asma, lo que conduce a una menor capacidad para realizar actividad física, predisponiendo a la obesidad (Wadden et al., 2018).

La obesidad en niños en edad escolar produce alteraciones mecánicas ventilatorias, debido a acumulaciones excesivas de masa adiposa (MA) a nivel abdominal. Esta acumulación abdominal está asociada a alteraciones biomecánicas y funcionales de la musculatura abdominal y diafragmática (Brashier \& Salvi, 2013), repercutiendo en las presiones generadas en etapas de inspiración y espiración forzada, alterando indicadores como la presión inspiratoria máxima (PIM) y presión espiratoria máxima (PEM) (da Jung \& Schivinski, 2014). Además, la inflamación en bajo grado inducida por obesidad puede predisponer a hiper-respuestas inflamatorias en el sistema respiratorio (Scott et al., 2017), aumentando la resistencia en la vía aérea, alterando flujos, presiones respiratorias y derivando en patrones respiratorios obstructivos (Sideleva et al., 2013). Estas alteraciones en fuerza muscular y flujos respiratorios se asocian con mayor frecuencia de sintomatología respiratoria y la aparición más frecuente y con mayor severidad de patologías respiratorias agudas (Okubo, 2017). Estas patologías destacan por su prevalencia y por ser una de las primeras causas de muerte infantil (Thompson et al., 2011).

Para el tratamiento de la OI se recomiendan abordajes interdisciplinarios al escolar y su familia, incluyendo apoyo nutricional, entrenamiento físico y acompañamiento psicológico para modificar los estilos de vida (García-Hermoso et al., 2018). Estas estrategias han demostrado ser eficaces en generar cambios en el índice de masa corporal (IMC) e IMCz Score (Al-Khudairy et al., 2017), composición corporal, perfil metabólico y estado inflamatorio del infante (García-Hermoso et al., 2018). El tratamiento nutricional tiene por obje- tivo educar sobre alimentación saludable y adecuar los niveles de ingesta a la actividad física realizada. El tratamiento psicológico busca consolidar los cambios de conducta y mejorar la adhesión al tratamiento(AlKhudairy et al., 2017).

El entrenamiento físico busca aumentar el gasto calórico semanal, permitiendo un mayor gasto de energía a partir de la MA y aumentando la masa muscular (MM) esquelética (Navarro et al., 2016). El tipo de entrenamiento recomendado para este objetivo es el entrenamiento concurrente, combinando trabajo de fuerza y aeróbico en sesiones intercaladas (GarcíaHermoso et al., 2018).

Actualmente no hay estudios que demuestren aumentos de PIM y PEM en escolares obesos sin patología respiratoria, producto de tratamientos disciplinares o interdisciplinares. Con estos antecedentes, la hipótesis del presente estudio es que una intervención interdisciplinar podría optimizar la mecánica ventilatoria, aumentando la PIM y PEM a través de las mejoras en el IMCz Score y asociado a cambios en la composición corporal. El objetivo de este estudio es analizar los efectos de un programa de intervención interdisciplinar con nutricionista, psicólogo y entrenador físico de tres meses de duración sobre el IMCz Score, la MA, MM y la fuerza muscular respiratoria (FMR) en escolares con OI y sin patología respiratoria.

\section{Material y métodos}

\section{Participantes}

El presente estudio es de tipo descriptivo de la situación de los individuos, en estudio previos y posterior a la intervención, con resultados absolutos y estandarizados a la referencia de la población en estudio, donde se reclutaron pacientes atendidos en el Hospital Doctor Gustavo Fricke, de Viña del Mar, Chile. Se seleccionó a escolares de entre 8 a 13 años de ambos sexos, con diagnóstico de OI. Se invitó a cada sujeto y a su familia a participar en este programa de estudio. De aceptar y cumplir con los criterios para participar, los tutores debía firmar el consentimiento informado y los escolares debieron aprobar su participación con el asentimiento informado. El estudio fue aprobado por el Comité de Ética de la Universidad Santo Tomás, Chile, código de aprobación 20.97, se hace patente el respeto por las normas éticas del trabajo con seres humanos de la Declaración de Helsinki y está de acuerdo a la Asociación Médica Mundial.

Los participantes debieron cumplir con los siguientes criterios de inclusión: 1) diagnóstico médico de 
obesidad con IMCz Score, según los patrones de crecimiento de escolares y adolescentes entre 5 años 1 mes y 19 años, definiendo la obesidad como los valores de IMC para la edad mayores a +2 desviaciones estándar sobre la referencia de la Organización Mundial de la Salud (OMS) (IMCz Score > 2) (Onis et al., 2007); 2) ser usuarios del Servicio de Salud Viña del Mar-Quillota, Chile. Los escolares fueron excluidos: 1) si presentaban patología cardiovascular que impidiera la práctica de actividad física; 2) si tenían un diagnóstico de asma u otra patología respiratoria crónica; y 3) un diagnóstico de intolerancia a la glucosa u otra patología metabólica.

\section{Procedimientos}

Toda la toma de datos se llevó a cabo en dependencias de la Universidad Santo Tomás, sede de Viña del mar, evaluando FMR y composición corporal, pre y post intervención.

\section{Fuerza Muscular Respiratoria}

La FMR se evaluó a través de la PIM y PEM en centímetros de agua $\left(\mathrm{cmH}_{2} \mathrm{O}\right)$, con un pimómetro portátil digital (MicroRPM ${ }^{\circledR}$, USA, Parkway). Las evaluaciones fueron realizadas acorde a los estándares y criterios de aceptabilidad de la declaración de la ATS para la función de la musculatura respiratoria (American Thoracic Society/European Respiratory Society, 2002). Los valores de PIM y PEM se expresaron en valores absolutos, en valores relativos al IMC y relativos a los valores predichos para la población chilena, según género, edad e IMC (Contreras et al., 2007).

\section{Composición Corporal}

Se utilizaron las medidas antropométricas del perfil restringido descritas por la ISAK (Marfell-Jones, M. et al., 2006) para la determinación de la composición corporal, se consideró el tejido adiposo y muscular utilizando las ecuaciones propuestas por Kerr (1988) para su modelo de 5 componentes. Las mediciones fueron realizadas por un evaluador certificado ISAK nivel II. La masa corporal fue evaluada con una balanza digital $\left(\mathrm{SECA}^{\circledR}\right.$ 813, Alemania Hamburgo) y el resto de las mediciones fueron realizadas con el kit antropométrico Rosscraft (Versión Gaucho pro ${ }^{\circledR}$, Buenos Aires Argentina).

\section{Programa de tratamiento interdisciplinario}

El programa interdisciplinario estuvo conformado por profesionales psicólogos, nutricionistas y entrenadores físicos. La interdisciplinariedad se logró a través de la coordinación permanente entre los profesionales participantes; se destaca que una vez a la semana se realizaron reuniones entre todas las disciplinas, coordinando las atenciones para optimizar el tratamiento. Los objetivos y funciones de cada rol fueron los siguientes:

\section{Atención psicológica}

Los psicólogos realizaron una entrevista inicial del programa donde se explicó y firmó el consentimiento y asentimiento informado. Una vez aceptada la participación, se evaluó la motivación al cambio mediante el cuestionario de estadios de cambio para el ejercicio físico (URICA-E2) en su versión validada al español (Moreno 2010), para luego asistir a seis talleres grupales de 60 minutos de duración, cada 15 días, en un periodo de tres meses. El objetivo de los talleres fue apoyar al escolar y a su familia en el proceso de rehabilitación, consolidando cambios de hábitos y mejorando la adhesión al tratamiento, mediante el abordaje de las temáticas: autoestima, preparación y voluntad al cambio, autopercepción, autocontrol, acoso escolar, adquisición de nuevos hábitos, entre otros.

\section{Atención nutricional}

El objetivo del tratamiento nutricional fue ajustar la ingesta a los requerimientos calóricos estimados para la actividad física del escolar, la etapa de desarrollo y crecimiento, además de educar sobre el consumo y preparación de alimentos saludables. El nutricionista aplicó un diagnóstico nutricional a través de la evaluación del IMC y su clasificación IMCz score al comienzo y al final del programa. Se confeccionaron recomendaciones dietéticas de acuerdo al diagnóstico nutricional y a los niveles de actividad física estimados para el usuario según las ecuaciones y referencias de Food and Nutrition Board (National Research Council. 2005). Se trabajó en sesiones cada 15 días con la familia y el usuario, logrando un total de 6 sesiones en 3 meses. En cada sesión se monitorizó la ingesta calórica diaria a través del recordatorio de 24 horas y encuestas de frecuencia de consumo.

\section{Protocolo de entrenamiento físico}

El entrenamiento físico fue supervisado por profesores de educación física y kinesiólogos. Se realizaron dos sesiones grupales de entrenamiento a la semana, de una hora de duración cada una, intercalando 48 a 72 horas entre las sesiones; cada sesión de ejercicio se realizó entre las 17 y 18 horas, los lunes y jueves, realizando 24 sesiones en 3 meses. Las estrategias de entre- 


\begin{tabular}{|c|c|c|c|c|c|c|c|c|c|c|c|}
\hline \multirow[t]{2}{*}{ a) } & & & \multicolumn{2}{|l|}{ DA } & \multicolumn{2}{|l|}{ DA } & \multicolumn{2}{|l|}{ DA } & \multicolumn{2}{|l|}{ DA } & \multirow[b]{2}{*}{ 5-10 min } \\
\hline & $5 \mathrm{~min}$ & $2-3 \min$ & $2 \mathrm{~min}$ & $2-3 \mathrm{~min}$ & $2 \mathrm{~min}$ & $2-3 \mathrm{~min}$ & $2 \mathrm{~min}$ & $2-3 \min$ & $2 \min$ & & \\
\hline \multirow{4}{*}{ b) } & EC & ES 1 & & El 1 & & ET & & ES 2 & & EI 2 & ve \\
\hline & & & & & & DA & & & & & \\
\hline & $5 \mathrm{~min}$ & \multicolumn{4}{|c|}{$15-20 \mathrm{~min}$} & $3-5 \min$ & \multicolumn{4}{|c|}{$15-20 \mathrm{~min}$} & 5-10 min \\
\hline & EC & \multicolumn{3}{|c|}{ AA 1} & & & \multicolumn{4}{|c|}{ AA 2} & vc \\
\hline
\end{tabular}

Figura 1. Descripción esquemática del protocolo de entrenamiento: a) circuito de fuerza resistencia; EC: calentamiento e instrucciones; ES 1 y ES 2: entrenamiento de extremidades superiores; El 1 y El 2: entrenamiento de extremidades inferiores; ET: entrenamiento de tronco; DA: descanso activo; VC: vuelta a la calma. b) circuito aeróbico; EC: calentamiento e instrucciones; AA 1 y AA 2: actividades aeróbicas; DA: descanso activo; VC: vuelta a la calma. Cada protocolo de entrenamiento se aplicó una vez por semana, en días no consecutivos.

namiento físico fueron: actividad aeróbica con juegos de rol competitivo y circuitos y trabajo de fuerza-resistencia de moderada intensidad en sesiones distintas.

Cada sesión comenzó con una explicación inicial del circuito y de los descansos y ejercicios a realizar. La entrada en calor (EC) consistió en dinámicas grupales lúdicas, como coreografías musicales infantiles. La intensidad se evaluó durante la estación mediante frecuencia cardiaca con un pulsómetro (Polar ft $1^{\circledR}$ ), manteniendo valores entre $60 \%$ a $80 \%$ de su frecuencia cardiaca de reserva, calculada con la fórmula de Karvonen (Karvonen \& Vuorimaa, 1988). Los descansos fueron activos a intensidades bajas, $40 \%$ a $60 \%$, durando 2 minutos entre series en el trabajo de fuerza y de 3 a 5 minutos durante el trabajo aeróbico. La vuelta a la calma (VC) consistió en ejercicios de relajación, elongaciones y ejercicios respiratorios.

El circuito de fuerza resistencia se aplicó según se detalla en la Figura 1. El entrenamiento de extremidades superiores se realizó con ejercicios de tipo Push up en diferentes angulaciones y cargas. El entrenamiento de extremidades inferiores, con ejercicios de tipo Squat, variando cargas y disposición de apoyos. El entrenamiento de tronco, con ejercicio de tipo abdominales, variando ángulos, cargas y apoyos. El trabajo aeróbico se realizó según se detalla en la Figura 1 b. Las actividades aeróbicas se realizaron con trabajo en grupos contra tiempo o competencia entre los grupos.

\section{Control médico}

Los usuarios asistieron a control médico con pediatría al inicio y después de la intervención. Los parámetros evaluados fueron: peso, talla, maduración sexual con escala Tanner según el protocolo descrito por Travers et al. (2014) y control de exámenes como perfil lipídico, electrocardiograma y ecografía.

\section{Análisis Estadístico}

El programa estadístico utilizado fue IBM SPSS Statistics $19^{\circledR}$ (Chicago, Estados Unidos). En primer lugar, se calcularon las medidas descriptivas de las variables objeto de estudio (promedio, mediana, desviación estándar e intervalos de confianza de la muestra); se incluye el coeficiente de variación para las variables de composición corporal y presiones respiratorias. Para analizar la distribución de la muestra, se utilizó la prueba de Shapiro-Wilk y el análisis gráfico de histogramas de frecuencia. Para el análisis inferencial, se utilizó un modelo t-test para datos pareados y se calculó el tamaño del efecto $(E S)$ de la intervención a través de d de Cohen ( $d$ ), clasificándolo según lo descrito por Cohen (Cohen, 1992). Para verificar asociaciones entre la FMR y la composición corporal, se utilizó Spearman $r$ Test. La significancia estadística que se utilizó en este estudio fue de $p<0.05$.

\section{Resultados}

Inicialmente se reclutaron 15 escolares, 4 abandonaron el estudio por falta de asistencia a las sesiones ( $\mathrm{Fi}$ gura 2). La muestra final fue de 11 escolares. Las características de los participantes se presentan en la Tabla 1.

En la muestra final, 7 escolares fueron de género masculino, 3 de ellos con una edad puberal Tanner I y 4 Tanner II, mientras que los 4 escolares restantes fueron de género femenino, 2 de ellas con una edad puberal Tanner II y 2 Tanner III. Previo a la intervención, todos los escolares se encontraban en obesidad (100\%) según IMCz Score para la edad. Post-intervención encontramos 5 individuos en sobrepeso (45.45 \%) y 6 en obesidad (54.55\%) según IMCz Score para la edad. Al analizar estadísticamente esta variable, se 
Tabla 1. Descripción del grupo de estudio.

\begin{tabular}{|c|c|c|c|c|c|c|c|c|}
\hline & \multicolumn{3}{|c|}{ Pre } & \multicolumn{3}{|c|}{ Post } & \multirow{2}{*}{$p$ Valor } & \multirow{2}{*}{ ES } \\
\hline & Promedio \pm DS & Mediana & IC & Promedio \pm DS & Mediana & IC & & \\
\hline Edad (años) & $10.18 \pm 1.53$ & 10 & $9-11$ & $10.3 \pm 1.65$ & 10 & $9-11$ & 0.49 & --- \\
\hline Peso $(k g)$ & $56.35 \pm 11.63$ & 61.5 & $48.54-64.17$ & $57.02 \pm 10.93$ & 62 & $49.67-64.36$ & 0.117 & 0.058 \\
\hline Talla $(\mathrm{cm})$ & $144.3 \pm 9.39$ & 141 & $138-150.6$ & $146 \pm 9.68$ & 141 & $139.5-152.5$ & $0.0042 * *$ & 0.178 \\
\hline IMC $\left(\mathrm{kg} / \mathrm{m}^{2}\right)$ & $26.8 \pm 3.4$ & 26.6 & $24.5-29.11$ & $26.58 \pm 3.39$ & 26,17 & $24.3-28.86$ & 0.16 & 0.065 \\
\hline IMCz Score & $2.17 \pm 0.232$ & 2.1 & $2.0-2.3$ & $2.073 \pm 0.293$ & 2 & $1.9-2.3$ & 0.04 * & 0.834 \\
\hline MM (\%) & $36.79 \pm 2.24$ & 37.14 & $34.71-38.87$ & $38.43 \pm 1.97$ & 37.72 & $36.61-40.26$ & 0.2188 & 0.777 \\
\hline MA (\%) & $36.31 \pm 2.88$ & 37.48 & $33.64-38.97$ & $34.21 \pm 2.87$ & 34.79 & $31.56-36.87$ & 0.078 & 0.73 \\
\hline
\end{tabular}

DS: Desviación estándar; IC: Intervalo de confianza 95\%; IMC: índice de masa corporal; IMCz Score: índice de masa relativo contrastado con datos de referencia OMS (Onis et al., 2007); MA: masa adiposa; MM: masa muscular; ES: tamaño del efecto. Datos representativos de 11 sujetos. * $p<0.05 * * p<0.01$. Wilcoxon matched-pairs signed rank test.

Tabla 2. Presiones respiratorias estandarizadas por IMC y valores de referencia población chilena (Contreras et al., 2007).

\begin{tabular}{|c|c|c|c|c|c|c|c|c|}
\hline & \multicolumn{3}{|c|}{ Pre } & \multicolumn{3}{|c|}{ Post } & \multirow{2}{*}{$\mathrm{p}$ Valor } & \multirow{2}{*}{ ES } \\
\hline & Promedio \pm DS & Mediana & IC & Promedio \pm DS & Mediana & IC & & \\
\hline PIM/IMC & $3.002 \pm 0.723$ & 2.843 & $2.516-3.488$ & $3.287 \pm 0.7613$ & 3.003 & $2.775-3.798$ & 0.021 * & 0.573 \\
\hline PEM/IMC & $3.145 \pm 0.8215$ & 3.164 & $2.593-3.697$ & $3.788 \pm 0.499$ & 3.865 & $3.45-4.124$ & $0.0015^{* *}$ & 0.643 \\
\hline PIM (\% referencia) & $77.44 \pm 16.66$ & 73.24 & $66.24-88.63$ & $83.8 \pm 14.87$ & 78.46 & $73.81-93.79$ & $0.0263 *$ & 0.754 \\
\hline PEM (\% referencia) & $62.14 \pm 15.31$ & 63.23 & $51.85-72.42$ & $74.48 \pm 8.927$ & 72,57 & $68.48-80.48$ & $0.0039 * *$ & 0.21 \\
\hline
\end{tabular}

DS: Desviación estándar; IC: Intervalo de confianza 95\%; IMC: índice de masa corporal; IMCz Score: índice de masa relativo contrastado con datos de referencia OMS (Onis et al., 2007); MA: masa adiposa; MM: masa muscular; ES: tamaño del efecto. Datos representativos de 11 sujetos. * $p<0.05 * * p<0.01$. Wilcoxon matched-pairs signed rank test.

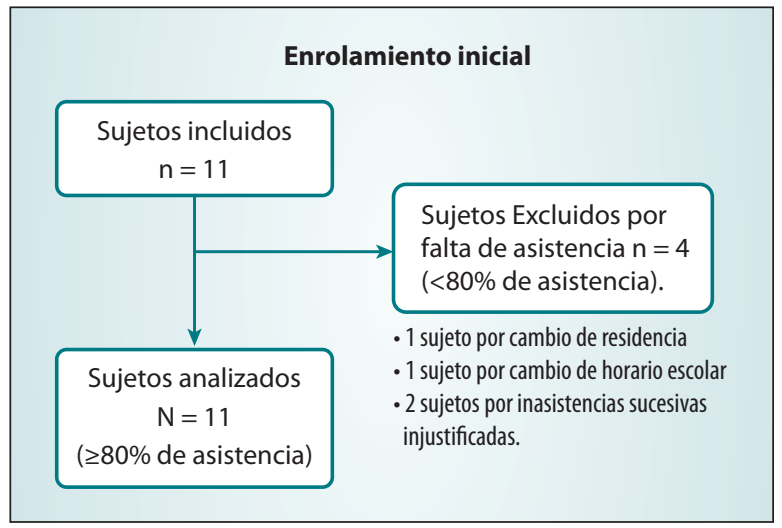

Figura 2. Diagrama de flujo de participantes del estudio y sus causas de exclusión.

encontró una disminución significativa de IMCz Score (Tabla 1). El IMC absoluto no mostró diferencias significativas entre la evaluación pre-intervención y la evaluación post (Tabla 1) (CV Pre: $12.8 \%$; Post: $12.75 \%$ ).

No se observaron cambios significativos en el peso total de los participantes después de la intervención. Sin embargo, la talla incrementó significativamente con un tamaño del efecto pequeño (Tabla 1). Además, se observó un descenso no significativo en la MA y no se registraron variaciones en la MM (Tabla 1 ).

En cuanto a la FMR, la PIM incrementó significativamente post-intervención (pre: $=79.27 \pm 17 \mathrm{cmH}_{2} \mathrm{O}$,
CV: 21.4\%; post: $85.82 \pm 15.01 \mathrm{cmH}_{2} \mathrm{O}, \mathrm{CV}: 17.5 \%$; $p$ $=0.029 E S=0.357$ ) (Figura 3a). De manera similar, la PEM incrementó significativamente post-intervención $\left(\right.$ pre $=83.18 \pm 19.94 \mathrm{cmH}_{2} \mathrm{O}$; post $=99.73 \pm 11.12$ $\mathrm{cmH}_{2} \mathrm{O} ; p=0.0064 ; E S=0.973$ ) (Figura $3 \mathrm{~b}$ ).

Se observó un aumento significativo de la PIM y la PEM tanto al relativizar sus valores con el crecimiento del individuo expresado por el IMC del momento de la evaluación, como al relativizarlas con los valores predichos de la población chilena, según peso, edad y género (Tabla 2).

En la asociación entre las variaciones de FMR y las variaciones de composición corporal, antes y después de la intervención, se observaron asociaciones significativas entre la PIM y MA (Figura 4a) y entre la PEM y MM (Figura 4b). Las asociaciones entre PIM con MM $(p=0.116 ; r=-0.501)$ y PEM con MA $(p=0.6187 ; r=$ $-0,169)$ no resultaron significativas.

\section{Discusión}

El objetivo del presente estudio fue analizar los efectos de un programa de intervención interdisciplinario de tres meses de duración sobre la MA, MM y la FMR de escolares con OI y sin patología respiratoria. Los principales hallazgos fueron: 1) un aumento significativo 


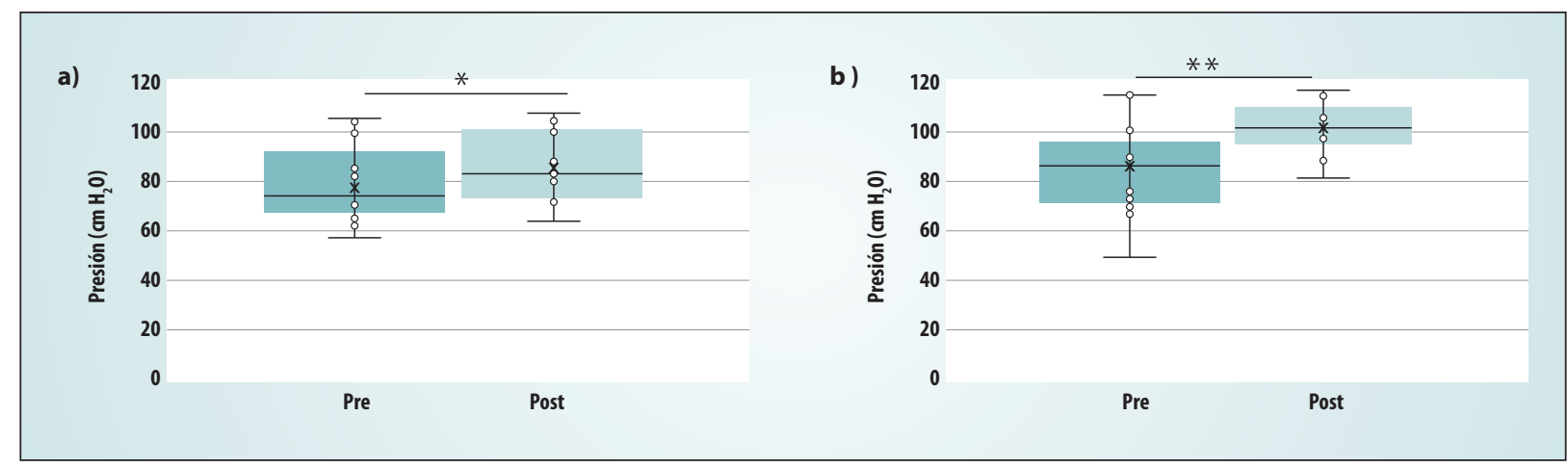

Figura 3. Variaciones de presiones respiratorias máximas, previo (Pre) y posterior (Post) a intervención de 3 meses. Número de sujetos $=11$. a) presión inspiratoria máxima (PIM); b) presión espiratoria máxima (PEM). Datos individuales representados en círculos abiertos. ${ }^{*} p<0.05 ; * * p<0.01$. Wilcoxon matched-pairs signed rank test.

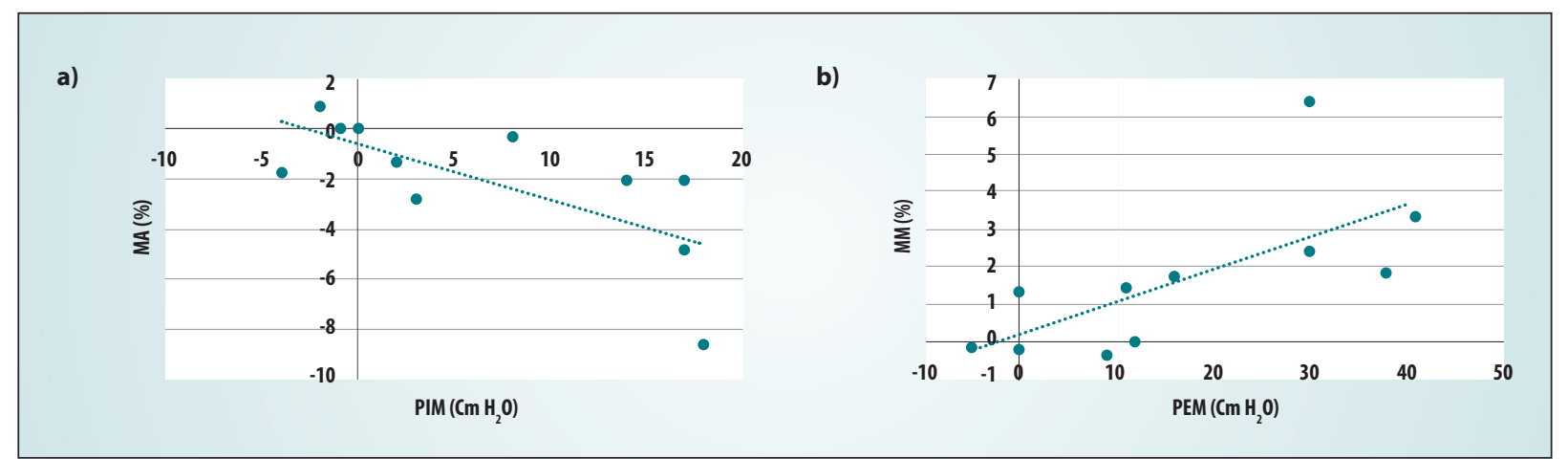

Figura 4. Asociaciones entre las variaciones de PIM y PEM con variables de composición corporal. a) Asociación entre PIM y masa adiposa (MA), $p=0.005$; $r=-0.769$. b) Asociación entre PEM y masa muscular (MM), datos individuales representados en círculos cerrados, $p=0.001 ; r=0.835$. Spearman $r$ test.

de la FMR expresadas en PIM y PEM; 2) disminución significativa de IMCz Score, sin variaciones significativas de la MA y MM.

Intervenciones similares han sido utilizadas en estudios previos, destacando por sus efectos a la hora de la prevención y el tratamiento de la OI, involucrando al escolar, su familia y entorno (Bahia et al., 2018; Nemet et al., 2005). En el presente estudio no podemos individualizar los resultados por disciplina, ya que cada profesional aportó a la rehabilitación del escolar y su entorno.

En variables antropométricas se observó un aumento significativo en la talla de los participantes, con un ES pequeño. El IMCz Score disminuyó significativamente, con un $E S$ alto. En relación con la composición corporal, no se observaron variaciones significativas y el ES fue moderado. De forma similar, Nemet et al. (2005) observaron mejoras en el IMCz Score, pero también mejoras en el peso y MA después de un programa de 3 meses de rehabilitación interdiciplinaria a escolares con entrenamientos presenciales 2 veces a la semana a través de juegos de equipos y juegos de carrera; a los escolares, además, se les pidió que a la semana acumularan 45 minutos de caminata a intensidad moderada. Como parte del estudio de Nemet et al se destaca que tanto nutricionistas como entrenadores prestaron especial atención en disminuir las conductas sedentarias de los escolares, programando sus actividades diarias. En revisiones y un metaanálisis de la evidencia, Bahía et al. (2018) y Whitlock et al. (2010) concluyeron que programas de 3 meses de duración pueden disminuir significativamente el $\mathrm{IMCz}$ Score, pero la evidencia de mejoras en la composición corporal en intervenciones interdisciplinarias basadas en entrenamiento físico o basadas en cambios de hábitos nutricionales menores a 6 meses es poca e insuficiente (Bahia et al., 2018; Whitlock et al., 2010). En el presente estudio no se encontraron diferencias significativas en la composición corporal de los sujetos; ello puede ser explicado porque la intervención de 3 meses de duración fue insuficiente o, tal como relatan Nemet et al, el no controlar las conductas sedentarias en el hogar puede que ralentice los cambios morfológicos.

El aumento significativo de la talla del individuo es un resultado esperable, ya que, por su edad y etapa de crecimiento, los escolares tienden a aumentar su talla rápidamente, generando desviaciones estándar mínimas y con comportamientos homogéneos. Sin embargo, se ve reflejado por un tamaño del efecto clasificado 
como mínimo. En el caso del peso corporal, el comportamiento es menos homogéneo; se observó que algunos individuos aumentan, otros disminuyen y algunos se mantienen, generando desviaciones mucho más amplias en el comportamiento de esta variable. Este comportamiento del peso podría explicar por qué no hay una variación significativa del IMC, generando un tamaño del efecto mínimo, pese a existir un aumento significativo de la talla. En el caso del IMCz Score, la tabla de referencia utilizada es la de OMS (Onis et al., 2007), la cual entrega referencias para la edad cada 3 meses. En el presente estudio todos los sujetos variaron su valor de referencia entre las evaluaciones pre y post. Es destacable que, inicialmente, según IMCz Score, teníamos a los 11 individuos clasificados como obesos, mientras que post-intervención, 5 de ellos pasaron a ser clasificados como sobrepeso. Este resultado puede estar explicando en parte el aumento significativo de la FMR, ya que la evidencia demuestra que hay mayores efectos respiratorios deletéreos en individuos obesos en comparación con los de sobrepeso (Forno et al., 2018; Wadden et al., 2018); por lo tanto, en los individuos que pasaron de OI a sobrepeso podemos esperar mejoras en la FMR.

En el presente estudio, al comparar las presiones respiratorias con sus valores de referencia para la población chilena (Contreras et al., 2007), observamos que antes de la intervención todos los usuarios tuvieron presiones inferiores a los valores de referencia. Utilizando la referencia para la población chilena se destaca el estudio de Rodríguez-Valdés et al (2019), quienes observaron resultados similares al analizar a 46 individuos con OI o sobrepeso, encontrando que sus presiones inspiratorias y espiratorias fueron significativamente menores a las de los individuos normopeso (Rodríguez-Valdés et al., 2019). Es importante destacar que, clínicamente, presiones respiratorias menores se relacionan con sensaciones de falta de aire durante la actividad física, y bajas en presiones espiratorias se asocian con tos inefectiva, problemas respiratorios obstructivos y mayor riesgo de patologías respiratorias infecciosas agudas (Furman et al., 2015), como la provocada por el Covid-19 (Sinha et al., 2020).

Este trabajo es el primero en evidenciar que tanto la PIM como la PEM de escolares obesos mejoran significativamente después de 3 meses de intervención, tanto en sus valores absolutos, relativos al IMC y relativos a los valores de referencia para la población chilena (Contreras et al., 2007). Además, es el primer estudio en observar que las variaciones significativas de la PIM se asocian indirectamente con los cambios de la
MA y las variaciones de la PEM se asocian directamente con los cambios observados en la MM. Asociaciones similares han sido observadas en estudios descriptivos de OI, como el de Kongkiattikul et al. (2015), quienes correlacionaron la capacidad residual funcional respiratoria con la MA. Una posible explicación la propone Magnani \& Cataneo (2007), quienes observaron que en adultos obesos existía una disminución significativa de las PIM y PEM, indicadores que aumentaban significativamente después de una cirugía de by-pass gástrico y una pérdida de peso corporal. Posiblemente el mecanismo involucrado es una disfunción en la musculatura respiratoria, producto de la sobrecarga de la pared torácica y alteración de la función contráctil muscular (Parreira et al., 2012), situación que se podría revertir con la disminución del IMCz Score. Navarro et al. (2016) observaron cómo 8 semanas de entrenamiento abdominal mejora significativamente la PEM en adolescentes sedentarios. Strongoli et al. (2010) demostraron que el entrenamiento de sentadillas y de extremidades superiores activa la musculatura respiratoria, aumentando las presiones transdiafragmáticas. En el presente estudio el entrenamiento físico incluyó trabajo de abdominales, extremidades superiores e inferiores, pudiendo mejorar la funcionalidad de la musculatura respiratoria, explicando, en parte, las variaciones en PIM y PEM encontradas en la presente investigación.

Para futuros estudios sería importante evidenciar si las mejoras en PIM y PEM se asocian a disminuciones de sintomatología respiratoria y a menor incidencia de patologías agudas respiratorias.

En el presente estudio no tenemos un grupo control con el cual comparar las variaciones observadas, lo cual representa una limitación. Pero es importante destacar que tanto el IMC como la FMR se estandarizaron de acuerdo a valores de referencia, validados para esta población, que consideran edad, peso y talla, por lo cual indirectamente pueden representar el comportamiento de individuos sin intervención. Pese a ello, para futuros estudios se sugiere incluir grupo control.

\section{Conclusiones}

Tres meses de tratamiento interdisciplinario de la obesidad infantil conducen a incrementos en las presiones espiratorias e inspiratorias máximas de los escolares evaluados y estos incrementos se asocian a variaciones en la composición corporal. 


\section{BIBLIOGRAFÍA}

Al-Khudairy, L., Loveman, E., Colquitt, J. L., Mead, E., Johnson, R. E. Fraser, H., ... Rees, K. (2017). Diet, physical activity and behavioural interventions for the treatment of overweight or obese adolescents aged 12 to 17 years. Cochrane Database of Systematic Reviews, 2017(6). https://doi.org/10.1002/14651858.CD012691

American Thoracic Society/European Respiratory Society. (2002). ATS/ ERS Statement on respiratory muscle testing. American Journal of Respiratory and Critical Care Medicine, 166(4), 518-624. https://doi. org/10.1164/rccm.166.4.518

Bahia, L., Schaan, C. W., Sparrenberger, K., Abreu, G. de A., Barufaldi, L. A., Coutinho, W., \& Schaan, B. D. (2018). Overview of meta-analyses on prevention and treatment of childhood obesity. Jornal de Pediatria, 95(4), 385-400. https://doi.org/10.1016/j.jped.2018.07.009

Brashier, B., \& Salvi, S. (2013). Obesity and Asthma: Physiological Perspective. Journal of Allergy, 2013, 1-11. https://doi. org/10.1155/2013/198068

Cohen, J. (1992). A Power Primer. Psychological Bulletin, 112(1), 155159. https://doi.org/10.1016/j.jorganchem.2011.01.025

Contreras, I., Caussade, S., Sanchez, I., Montalvo, D., Pineda, N., Fierro, L., \& Holmgren, N. (2007). Valores Espirométricos Normales En Niños Y Adolescentes Chilenos: Comparación Con Valores Extranjeros. Neumología Pediátrica, 8(153), 15-18.

da Jung, R. G., \& Schivinski, C. I. S. (2014). Avaliação da força muscular respiratória de crianças segundo a classificação do índice de massa corporal. Revista Paulista de Pediatria, 32(2), 250-255. https://doi. org/10.1590/0103-0582201432210313

Forno, E., Han, Y. Y., Mullen, J., \& Celedón, J. C. (2018). Overweight, Obesity, and Lung Function in Children and Adults-A Meta-analysis. Journal of Allergy and Clinical Immunology: In Practice, 6(2), 570581.e10. https://doi.org/10.1016/j.jaip.2017.07.010

Furman, E. G., Yarulina, A. M., \& Sofronova, L. V. (2015). Lung Function and Respiratory Muscle Strength in Obese in Children. Pediatric Pharmacology, 12(2), 143. https://doi.org/10.15690/pf.v12i2/1276

García-Hermoso, A., Ramírez-Vélez, R., Ramírez-Campillo, R., Peter son, M. D., \& Martínez-Vizcaíno, V. (2018). Concurrent aerobic plus resistance exercise versus aerobic exercise alone to improve health outcomes in paediatric obesity: a systematic review and meta-analysis. British Journal of Sports Medicine, 52(3), 161-166. https://doi. org/10.1136/bjsports-2016-096605

GBD 2015 Obesity Collaborators, Afshin, A., Forouzanfar, M. H., Reitsma, M. B., Sur, P., Estep, K., ... Murray, C. J. L. (2017). Health Effects of Overweight and Obesity in 195 Countries over 25 Years. The New England Journal of Medicine, 377(1), 13-27. https://doi.org/10.1056/ NEJMoa1614362

Karvonen, J., \& Vuorimaa, T. (1988). Heart Rate and Exercise Intensity During Sports Activities Practical Application. Sports Medicine (Auckland, N.Z.), 312, 303-311.

Kerr, D. A. (1988). An anthropometric method for fractionation of skin, adipose, bone, muscle and residual tissue masses in males and females age 6 to 77 years (Doctoral dissertation, Theses (School of Kinesiology)/Simon Fraser University).

Kongkiattikul, L., Sritippayawan, S., \& Chomtho, S. (2015). Relationship between Obesity Indices and Pulmonary Function Parameters in Obese Thai Children and Adolescents. The Indian Journal of Pediatrics, 82(12), 1112-1116. https://doi.org/10.1007/s12098-015-1777-4

Magnani, K. L., \& Cataneo, A. J. M. (2007). Respiratory muscle strength in obese individuals and influence of upper-body fat distribution. Sao Paulo Medical Journal, 125(4), 215-219.

Marfell-Jones, M., Stewart, A., \& Olds, T. (2006). Kinanthropometry IX Proceedings of the 9th International Conference of the International Society for the Advancement of Kinanthropometry (Routledge., ed.)

Moreno, B. (2010). Estudio correlacional entre estadios de cambio para el ejercicio físico, motivación, intención de ser físicamente activo y fluidez disposicional en adolescentes extremenos [trabajo fin de Más- ter universitario en Investigación en Ciencias Sociales y Jurídicas con especialidad en Ciencias de la Actividad Física y el Deporte]. Trabajo Fin de Máster Universitario en Investigación en Ciencias Sociales y Jurídicas con especialidad en Ciencias de la Actividad Física y el Deporte. Universidad de Extremadura. Cáceres.

National Research Council. (2005). Food and Nutrition Board. Dietary reference intakes for energy, carbohydrate, fiber, fat, fatty acids, cholesterol, protein, and amino acids (macronutrients).

Navarro, X., Gatica, D., \& Manterola, C. (2016). Effect of abdominal muscle training on respiratory muscle strength and forced expiratory flows in sedentary, healthy adolescents. Archivos Argentinos de Pediatria, 114(5), 434-440. https://doi.org/10.5546/aap.2016.eng.434

Nemet, D., Barkan, S., Epstein, Y., Friedland, O., Kowen, G., \& Eliakim, A. (2005). Short- and Long-Term Beneficial Effects of a Combined Dietary-Behavioral-Physical Activity Intervention for the Treatment of Childhood Obesity. Pediatrics, 115(4), e443-e449. https://doi. org/10.1542/peds.2004-2172

Okubo, Y., Nochioka, K., \& Testa, M. A. (2017). The impact of pediatric obesity on hospitalized children with lower respiratory tract infections in the United States. The clinical respiratory journal, 12(4), 14791484. https://doi.org/10.1111/crj.12694

Onis, M. De, Onyango, A. W., Borghi, E., Siyam, A., \& Siekmann, J. (2007). Development of a WHO growth reference for school-aged children and adolescents. Bulletin of the World Health Organization, 043497(April), 660-667. https://doi.org/10.2471/BLT.

Parreira, V. F., Matos, C. M., Athayde, F. T., Moraes, K. S., Barbosa, M. H., \& Britto, R. R. (2012). Evolution of respiratory muscle strength in postoperative gastroplasty. Brazilian Journal of Physical Therapy, 16(3), 225-230. http://dx.doi.org/10.1590/S1413-35552012000300008

Rodríguez-Valdés, S., Donoso-Riveros, D., Sánchez-Peña, E., MuñozCofré, R., Conei, D., del-Sol, M., y Cabello, M.-E. (2019). Uso del Índice de Masa Corporal y Porcentaje de Grasa Corporal en el Análisis de la Función Pulmonar. International Journal of Morphology, 37(2), 592-599. https://doi.org/10.4067/s0717-95022019000200592

Scott, H. A., Wood, L. G., \& Gibson, P. G. (2017). Role of Obesity in Asthma: Mechanisms and Management Strategies. Current Allergy and Asthma Reports, 17(8). https://doi.org/10.1007/s11882-017-0719-9

Sideleva, O., Black, K., \& Dixon, A. E. (2013). Effects of obesity and weight loss on airway physiology and inflammation in asthma. Pulmonary Pharmacology and Therapeutics, 26(4), 455-458. https://doi. org/10.1016/j.pupt.2012.05.002

Sinha, I. P., Harwood, R., Semple, M. G., Hawcutt, D. B., Thursfield, R., Narayan, O., ... \& Southern, K. W. (2020). Covid-19 infection in children. The Lancet Respiratory Medicine, 8(5), 446-447. https://doi. org/10.1056/NEJMc2005073

Strongoli, L. M., Gomez, C. L., \& Coast, J. R. (2010). The effect of core exercises on transdiaphragmatic pressure. Journal of Sports Science and Medicine, 9(2), 270-274.

Thompson, W. W., Shay, D. K., Weintraub, E., Brammer, L., Cox, N., \& Anderson, L. J. (2011). Mortality associated with influenza and respiratory syncytial virus in the United States. Medicine, 289(2), 179186. doi:10.1001/jama.289.2.179

Travers, S. H., Jeffers, B. W., Bloch, C. A., Hill, J. O., \& Eckel, R. H. (1995). Gender and Tanner stage differences in body composition and insulin sensitivity in early pubertal children. The Journal of Clinical Endocrinology \& Metabolism, 80(1), 172-178.

Wadden, D., Allwood Newhook, L.-A., Twells, L., Farrell, J., \& Gao, Z (2018). Sex-Specific Association between Childhood BMI Trajectories and Asthma Phenotypes. International Journal of Pediatrics, 2018, 1-9. https://doi.org/10.1155/2018/9057435

Whitlock, E. P., O'Connor, E. A., Williams, S. B., Beil, T. L., \& Lutz, K. W. (2010). Effectiveness of Weight Management Interventions in Children: A Targeted Systematic Review for the USPSTF. Pediatrics, 125(2), e396-e418. https://doi.org/10.1542/peds.2009-1955 